\title{
Tempering Macrophage plasticity for controlling SARS-CoV-2 infection for managing COVID-19 disease
}

Devinder Toor ${ }^{1}$, Aklank Jain ${ }^{2}$, Shivani Kalhan ${ }^{3}$, Harmesh Manocha ${ }^{4}$, Vivek Kumar Sharma ${ }^{5}$, Payal Jain ${ }^{6}$, Vishwas Tripathi ${ }^{7}$ and Hridayesh Prakash ${ }^{1 *}$

1. Amity Institute of Virology and Immunology, Amity University Uttar Pradesh, Sector 125, Noida, Uttar Pradesh, India

2. Department of Zoology, Central University of Punjab, Bathinda, Punjab, India

3. Department of Pathology, Government Institute of Medical Sciences, Greater NOIDA, UP, India

4. Department of Microbiology Government Institute of Medical Sciences, Greater NOIDA, UP, India

5. Department of Physiology, Government Institute of Medical Sciences, Greater Noida, UP, India

6. Department of Medicine, Government Institute of Medical Sciences, Greater Noida, UP, India

7. School of Biotechnology, Gautam Buddha University, Greater Noida, 201308,GautamBudh Nagar, U.P.(India)

Running title: Macrophage plasticity and COVID-19

* Address for correspondence

Dr. Hridayesh Prakash

Amity Institute of Virology and Immunology,

Amity University Uttar Pradesh,

Sector-125, Noida, India

Phone: +91-120-4392961

Email: hprakash@amity.edu 


\begin{abstract}
Hyper activation of macrophages contributes to acute respiratory distress syndrome, respiratory failure, and subsequent death of COVID-19 cases. Given this, tempering macrophage plasticity is paramount and the highest priority for the management of COVID-19 cases. In this context we here propose that either exchange or in situ reprogramming of derailed Th17+ alveolar macrophages/ Slan+ DC with Th1 programmed counterpart would potentially mitigate or abolish pulmonary fibrosis. This approach is also anticipated to afford antiviral immune response and promote recovery in the patients and hold tremendous potential for managing severely infected patients by both curbing viruses and enhancing post-treatment recovery.
\end{abstract}




\section{Background}

The recent outbreak of the COVID-19 has challenged the health care system for developing effective and immediate therapeutics for controlling novel SARS-CoV-2 for the effective management of COVID-19 pandemic which is manifested by aberrant immunopathology. This is a new pathology and our knowledge about this disease is limited therefore lot of work is required before any therapeutics/vaccine could be launch for its eradication. Several recent and compelling studies have propose several aspects of pathological basis of disease (1) which enhanced our understanding how SARS-CoV2 is interacting with alveolar and peripheral tissues and causing the death of the infected patients.

\section{Role of macrophage in Pathogenesis of Covid 19}

SARS-CoV-2 induces pathogenic inflammation, pulmonary fibrosis, acute respiratory distress syndrome (ARDS) and nephropathy which ultimately causing the death of infected patients within 2-3 weeks (1). Hyper-activation of committed macrophages which are termed as macrophage activation syndrome (MAS) (2) is associated with ARDS (3) in manifesting respiratory failure and subsequent death of COVID-19 patients. Autopsy reports and single-cell RNA sequencing (4) revealed the presence of monocyte-derived $\mathrm{FCN}^{+}{ }^{+}$macrophages and other immune cells $(4 ; 5)$ in the broncho alveolar lavage fluid (BALF) of COVID-19 patients with severe ARDS. However due to abundance of macrophages over other immune cells and MAS in COVID 19 patients, these cells seems to be important for pathogenesis.

Transcriptional analysis of BALF further revealed high levels of IFN- $r$ induced protein-10 (IP-10) and various chemokines like MCP-1, CCL2, and CCL7 in BALF of severe COVID-19 patients which correlated with increased infiltration of monocyte / Macrophages (6) in their lungs. Cytokine profiling of critical COVID-19 patients reflects MAS with high levels of pro-inflammatory cytokines and chemokines such as IL-6, IL-7, Tumour Necrosis Factor (TNF- $\alpha$ ), CCL2, CCL3, CXCL10, and IL-2 receptor. This demonstrate dysregulated activation of the mononuclear phagocyte system, hyperinflammatory response (7) and MAS (8) which is causing death of patients. Based on 
these compelling evidence tempering macrophage plasticity seems to be one of the most suitable interventions for effective management of COVID-19 disease.

Both activation and increased infiltration of monocytes, dendritic cells, neutrophils, and lymphocytes (9) during infection leads to ARDS and cytokine storm in COVID-19 patients. During SARS-CoV-2 infection, activated CD4+ $\mathrm{T}$ lymphocytes also secrete granulocyte-macrophage colony-stimulating factor which further stimulates macrophage to secrete pro-inflammatory cytokines thereby continuing the vicious cycle of the cytokine storm. SARS-CoV-2 activate alveolar, splenic and renal macrophages through angiotensin-converting enzyme 2 (ACE2) and enhance the secretion of IL-6, TNF- $\alpha$, IL-10, and PD-1 (9) in patients. Both ARDS and MAS contribute to significant depletion of CD8+ CTLs which associated with disease severity (10) in COVID-19 patients. SARS-CoV-2 infects type 2 alveolar cells, epithelial cells and podocytes in lungs and kidney respectively and interacts with them via ACE2 receptors which facilitate the attachment and entry of this virus into host cells $(11 ; 12)$. ACE-2 / Angiotensin-II receptors are known to activate Sphingosine-1-phosphate receptor 1 (S$1 \mathrm{P}$ receptor 1) which is known to mediate IL-6 induced myopathy and fibrosis (13) S-1P R-1 signalling is associated with Th2 / 17 responses (14;15), hypoxia and allergic manifestations (16) which altogether promotes Th2 bias in the infected patients. Therefore it is rationale to presume that S-1P receptor 1 signalling would enhance the pathogenesis of COVID-19 cases. In view of this, blocking S-1P signalling either by application of S-1P lyase;(17) or FTY-720 / Fingolimod (18;19) seems rationale to modulate the pathogenesis of novel COVID-19 disease. In view of this FTY-720 is being explored in the Phase-2 clinical trial against COVID-19 patients (NCT04280588, MRCTA, ECFAH of FMU) and results are still awaited. However, due to its immunosuppressive nature, FTY-720 is expected to only lower down the hyperinflammatory response and may afford only symptomatic relief in COVID-19 cases. Thus this may not afford clearance of SARS-CoV-2 infection (20) in COVID-19 cases.

\section{Pre-clinical / Animal models for validation of proposed concept}

Like any other new proposed interventions, validating new hypothesis in pre-clinical model is essential before its clinical application in the patients. Depending upon mouse 
specific SARS-CoV-2 isolates, inbred mice like Balb/c \& C57BL/6 represent suitable model of testing vaccines, antiviral drugs and disease pathogenesis (21). Furthermore due to dependency of SARS-CoV-2 on Ace-2(22), Tmprss2 (23) and Stat1 (24) protein for manifesting disease, knockout mice of these proteins also represent a potential model for validating concept against COVID-19 disease. Most interestingly, floxed strain of these mice could be used and backcrossed with LysM Cre mice to address macrophages related phenotype. To demonstrate anti-Covid 19 potential of M1 macrophages, most suitable strategy is to either use clinical isolate of SARS-CoV-2 or express major spike proteins of this virus in VeroE6 cell line and co culture them with THP / CD14+ / CD11b+ macrophages and evaluate the influence of various adjuvants mentioned in Table -1 / Figure 1 on innate and adaptive immune response of both macrophages and CD8+CTL. Several aspects like maturation, M1 / Th1 programming of macrophages and subsequent killing of Virus / expression of Spike proteins can be used to correlate the M1 programming of RAW / THP / CD14+ / CD11b+ primary macrophages. Likewise $T$ cell response could be measured by co culturing $T$ cells with infected epithelial / macrophages co culture. Various parameters like TARC/CCL17, RANTES STAT-1, 6, calcineurin, IRF - 4, 8 factors Th1 / Th17 response could be evaluated for addressing the impact of $\mathrm{M} 1$ programmed macrophage on $\mathrm{T}$ cell activation and killing of infected epithelial cells. These per-clinical experiments are essential for validating anti-Covid impact of M1 / CD8+CTL. These approaches certainly represent most suitable approaches for validating the concept at pre-clinical levels and need further investigations

\section{Translational Approach}

In view of above facts, mitigating pathogenic inflammation (Th2/17 responses) and pulmonary fibrosis is a paramount requirement for the management of COVID-19 infection. In this context our previous studies have potentially demonstrated the significance of iNOS+M1 effector macrophages and CD8+CTL in controlling respiratory pathogens (6-8), which are associated with community-acquired pneumonia. On the basis of these studies, we believe that tweaking these effector immune cells may be sufficient to control replication of SARS-CoV2 infection as well in COVID-19 cases. This 
can be afforded either by their in situ reprogramming of Th2/ Th17 programmed macrophages or exchanging Th2/17 programmed macrophage with Th1 primed iNOS+CD14+M1 macrophages and CD8+CTL's in neo-adjuvant setting. Based on this and our previously published studies $(9 ; 10)$ we also believe that the exchange of Th17+ macrophages with Th1+ M1 macrophages may also afford protective immunity against SARS-CoV-2 infection in Covid 19 cases. This approach is anticipated to change the fate of severely infected patients but needs to be commissioned before its clinical application.

\section{Proposed intervention}

While the application of antiviral drugs like Remedesvir, Tocilizumab is sufficient to control the infection. Whether these drugs are adequate to augment immunogenic inflammation and clear infected cells from the infected organ is questionable. In this context, we have proposed the potential role of Ceramide- 1 phosphate as immune adjuvants for promoting Th1 programming of infected cells/ tissue and subsequent clearance of viral infected cells from the tissue (currently under revision). In view of potential involvement of S1P in fibrosis and Th1 bias, FTY720 based interventions is currently under Phase- $2^{\text {nd }}$ clinical trial (NCT04280588) which is based on trapping effector $\mathrm{T}$ cell in the lymph node for effective control of infection and mitigating cytokine storm. However due to immunosuppressive nature, we believe that FTY-720 may not afford help to the patients. Likewise other studies have also highlighted the significance of BCG vaccination with increased resistant to the infection (25) and demonstrated reduced mortality in dexamethasone treated (26) COVID 19 patients. Since these interventions are targeted to reduce inflammatory score and anticipated to provide only symptomatic relief therefore other more robust and stable interventions are required for managing disease.

Moreover several reports mentioned above have provided compelling evidence that hyper activated macrophages are critical for ARDS and subsequent death in the patients so considering these aspects, combining macrophage directed strategies with other available approaches (as adjunct approach) is decisive for effective management of severely infected cases thus certainly deserve attention. Out of several available 
options, most relevant strategy would be to promote in situ reprogramming of Th2/17 macrophages toward M1 by employing various neo adjuvants which are summarized in Table-1

In past we have identified several neo adjuvants like PDE4b/ AC mimetic which are capable of modulating Th17 response in human CD14+ monocyte and Slan DC (27), which complies with one of the recent study (3) which highlighted the potential of androgenic receptors in modulating cytokine storm. Therefore, we feel that PDE4/AC modulators (28) can also potentially modulate/mitigate macrophage activation syndrome and afford help in COVID-19 cases.

Apart from these, we have validated several other immune adjuvants like low does Gamma Radiation (29;30), S-1P (31), and Smac mimetics (32) bearing potential of Th1 programming of macrophages in pre-clinical models system. Therefore we believe that these adjuvants are likely to afford immunity against SARS-CoV-2 infection as well as in available animal models which we have discussed above. Inclusion of various other adjuvants mentioned in Table-1 targeting refractory macrophages, can also be potentially utilized for tempering macrophages plasticity for controlling SARS-CoV-2 infection effectively in COVID 19 cases.

\section{Macrophages/ MSC as palliative regimes for COVID 19}

Since macrophages are fairly plastic component of the body and could afford help for tissue regeneration and homeostasis after completion of therapy. These aspects are critical for the expected outcome of proposed immune / pharmacological interventions against infection. Apart from this, M2 or refractory macrophages also known to promote activity of fibroblast / mesenchymal stem cells/fibroblast $(33 ; 34)$ which can potentially neutralize therapy related inevitable adverse impacts. Both MSC and macrophages secrete various factors $(35 ; 36)$ which are involved in wound healing. On account of regenerative potential of macrophages $(37 ; 38)$ and their close association with mesenchymal stem cells $(39 ; 40)$ these cells can potentially be utilized as palliative approach $(41 ; 42)$ for accelerated recovery of patients. This certainly warrants the use of 
macrophages / MSC as whole-cell infusions or their products as palliative components for enhancing recovery of COVID 19 patients post interventions.

\section{Major Perspective}

The proposed interventions which is depicted in Figure 1 seem to be the candid approach for tuning pathogenic inflammation, Th1 reprogramming of derailed Macrophages, enhancing MHC-I dependent presentation of viral antigens to CD8+CTL for augmenting required adaptive immunity of host against SARS-CoV-2 infection. This is a novel immunotherapeutic approach and translationally viable in nature. The approach harbour potential for effective management of SARS-CoV-2 infection in severely infected COVID-19 patients. We also envisage that such an approach could be employed as potential adjunct to placid / antiviral therapies for effectively manage COVID 19 disease (both prospective and therapeutic in patients) thus certainly deserve immediate investigation for its clinical efficacy.

\section{Conflict of Interest:}

The author declare that they do not have any conflict of interest which need to be declared

\section{Author Contributions}

$\mathrm{HP}$, conceived the idea and supervised the entire study.

DT, AKJ, SK,HM, VS, contributed to research tool

DT and VT: Critical analysis

$\mathrm{HP}$ and AJ wrote the manuscript.

\section{Acknowledgment}

This work was supported by the Extramural funding from Department of Biotechnology BT/PR8282/MED/29/722/2013to HP. The funder had no role to play indecision-making process 


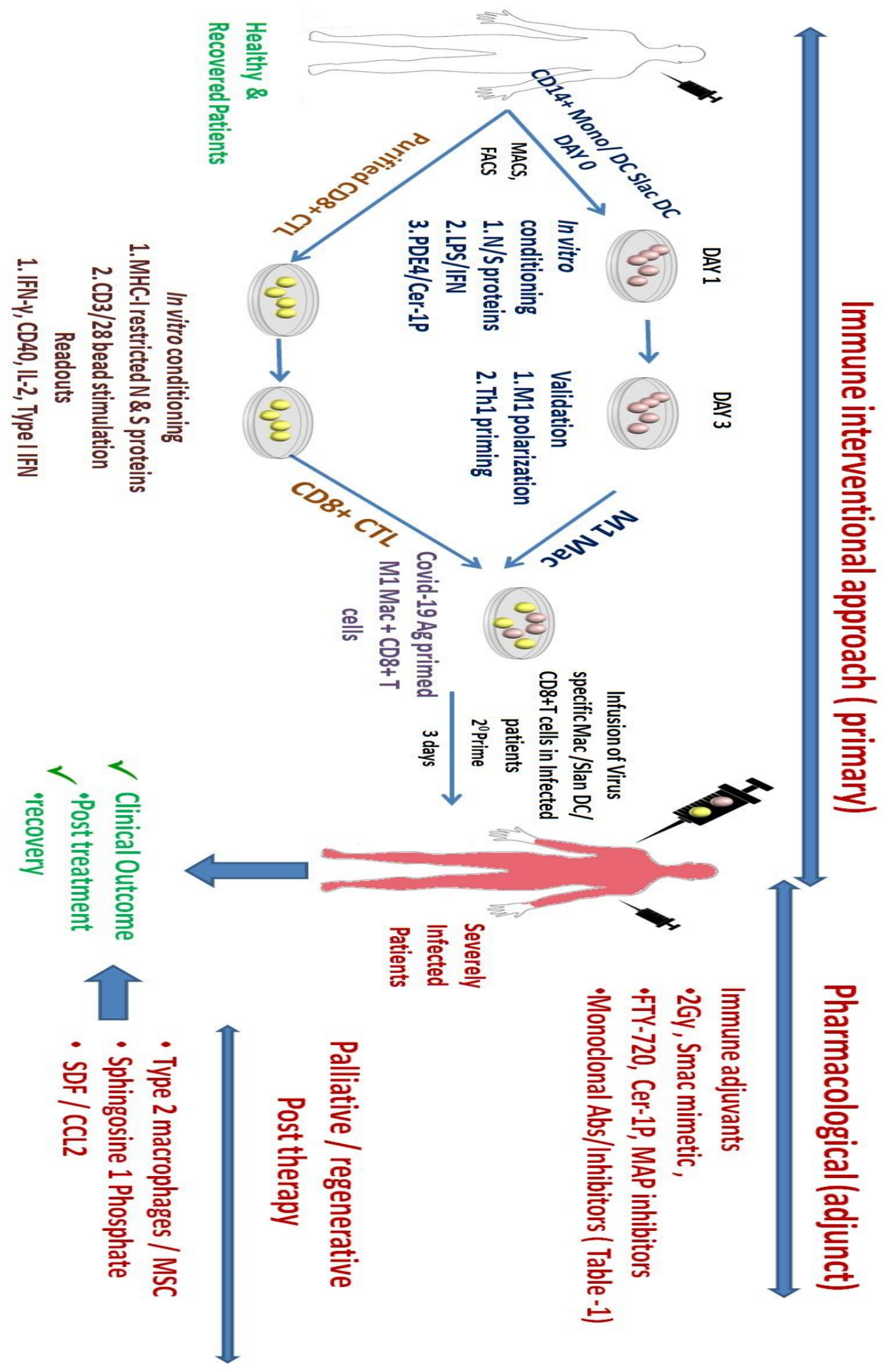


Figure 1: Schematic representation of augmenting SARS-Co-V2 specific adaptive immunity

Shown here are the most potent immune mediate (perspective) and/or pharmaceutical / palliative approaches for augmenting adaptive immune responses of derailed Macrophages / SlanDC / CD8+ T cells for the treatment and recovery of COVID-19 patients. These approaches are paramount requirement (as conjunction therapies) and expected to afford help for managing Covid 19 disease. 
Table 1

Clinical trials targeting Activated macrophages for indicated inflammatory and tumor Diseases

\begin{tabular}{|c|c|c|c|c|c|}
\hline Clinical Trials & $\begin{array}{c}\text { TAM } \\
\text { Targeting } \\
\text { agent }\end{array}$ & Target & $\begin{array}{l}\text { Clinical } \\
\text { Phase }\end{array}$ & Disease & Impact \\
\hline NCT03123783 & APX005M & CD40 & I/II/Active & NSCLC & $\begin{array}{l}\text { Macrophages } \\
\text { activation }\end{array}$ \\
\hline NCT01433172 & $\begin{array}{l}\text { (GM.CD40L) } \\
\text { vaccine with } \\
\text { CCL21 }\end{array}$ & CD40 & I/II/Active & Lung cancer & $\begin{array}{l}\text { Targeting } \\
\text { CD14+/CD16+ } \\
\text { alveolar } \\
\text { macrophages }\end{array}$ \\
\hline NCT02637531 & IPI-549 & PI3Ky & I/ Active & $\begin{array}{l}\text { Advanced solid } \\
\text { tumor; non- } \\
\text { small cell lung } \\
\text { cancer; } \\
\text { melanoma; } \\
\text { breast cancer }\end{array}$ & $\begin{array}{l}\text { Macrophage } \\
\text { plasticity }\end{array}$ \\
\hline NCT00397826 & Simvastine & p38MAPK & II/Active & COPD & $\begin{array}{l}\text { Macrophages } \\
\text { activation }\end{array}$ \\
\hline NCT04280588 & $\begin{array}{c}\text { FTY 720 } \\
\text { (Fingolimod) }\end{array}$ & S1P & II/Active & $\begin{array}{l}\text { COPD, Multiple } \\
\text { sclerosis }\end{array}$ & $\begin{array}{c}\text { proposed as a } \\
\text { prophylactic agent } \\
\text { against Covid19 }\end{array}$ \\
\hline NCT01103635 & ICINCB7839 & ADAM17 & I/ Active & Liver Cirrhosis & $\begin{array}{c}\text { Dampening Kuffer } \\
\text { cells activation }\end{array}$ \\
\hline NCT04261075 & $\begin{array}{l}\text { IPH5201 and } \\
\text { IPH5301 }\end{array}$ & $\begin{array}{l}\text { CD39 \& } \\
\text { CD73 }\end{array}$ & I & Cancer & $\begin{array}{c}\text { Activation of } \\
\text { macrophages and } \mathrm{T} \\
\text { cells }\end{array}$ \\
\hline
\end{tabular}




\section{Reference}

(1) Zhou F, Yu T, Du R, Fan G, Liu Y, Liu Z, et al. Clinical course and risk factors for mortality of adult inpatients with COVID-19 in Wuhan, China: a retrospective cohort study. Lancet 2020 Mar 28;395(10229):1054-62.

(2) Giamarellos-Bourboulis EJ, Netea MG, Rovina N, Akinosoglou K, Antoniadou A, Antonakos N, et al. Complex Immune Dysregulation in COVID-19 Patients with Severe Respiratory Failure. Cell Host Microbe 2020 Apr 17.

(3) Konig MF, Powell M, Staedtke V, Bai RY, Thomas DL, Fischer N, et al. Preventing cytokine storm syndrome in COVID-19 using alpha-1 adrenergic receptor antagonists. J Clin Invest 2020 Jul 1;130(7):3345-7.

(4) Liao M, Liu Y, Yuan J, Wen Y, Xu G, Zhao J, et al. Single-cell landscape of bronchoalveolar immune cells in patients with COVID-19. Nat Med 2020 Jun;26(6):842-4.

(5) Yao XH, Li TY, He ZC, Ping YF, Liu HW, Yu SC, et al. [A pathological report of three COVID-19 cases by minimal invasive autopsies]. Zhonghua Bing Li Xue Za Zhi 2020 May 8;49(5):411-7.

(6) Xiong Y, Liu Y, Cao L, Wang D, Guo M, Jiang A, et al. Transcriptomic characteristics of bronchoalveolar lavage fluid and peripheral blood mononuclear cells in COVID-19 patients. Emerg Microbes Infect 2020 Dec;9(1):761-70.

(7) Mehta P, McAuley DF, Brown M, Sanchez E, Tattersall RS, Manson JJ. COVID-19: consider cytokine storm syndromes and immunosuppression. Lancet 2020 Mar 28;395(10229):1033-4.

(8) Schulert GS, Grom AA. Pathogenesis of macrophage activation syndrome and potential for cytokinedirected therapies. Annu Rev Med 2015;66:145-59.

(9) Wang C, Xie J, Zhao L, Fei X, Zhang H, Tan Y, et al. Alveolar macrophage dysfunction and cytokine storm in the pathogenesis of two severe COVID-19 patients. EBioMedicine 2020 Jul;57:102833.

(10) Luo M, Liu J, Jiang W, Yue S, Liu H, Wei S. IL-6 and CD8+ T cell counts combined are an early predictor of in-hospital mortality of patients with COVID-19. JCI Insight 2020 Jul 9;5(13).

(11) Wrapp D, Wang N, Corbett KS, Goldsmith JA, Hsieh CL, Abiona O, et al. Cryo-EM structure of the 2019nCoV spike in the prefusion conformation. Science 2020 Mar 13;367(6483):1260-3.

(12) Hoffmann M, Kleine-Weber H, Schroeder S, Kruger N, Herrler T, Erichsen S, et al. SARS-CoV-2 Cell Entry Depends on ACE2 and TMPRSS2 and Is Blocked by a Clinically Proven Protease Inhibitor. Cell 2020 Mar 4.

(13) Ohkura SI, Usui S, Takashima SI, Takuwa N, Yoshioka K, Okamoto Y, et al. Augmented sphingosine 1 phosphate receptor-1 signaling in cardiac fibroblasts induces cardiac hypertrophy and fibrosis through angiotensin II and interleukin-6. PLoS One 2017;12(8):e0182329.

(14) Schroder M, Richter C, Juan MH, Maltusch K, Giegold O, Quintini G, et al. The sphingosine kinase 1 and S1P1 axis specifically counteracts LPS-induced IL-12p70 production in immune cells of the spleen. Mol Immunol 2011 May;48(9-10):1139-48.

(15) Schulze T, Golfier S, Tabeling C, Rabel K, Graler MH, Witzenrath M, et al. Sphingosine-1-phospate receptor $4(\mathrm{~S} 1 \mathrm{P}(4))$ deficiency profoundly affects dendritic cell function and TH17-cell differentiation in a murine model. FASEB J 2011 Nov;25(11):4024-36. 
(16) Saluja R, Kumar A, Jain M, Goel SK, Jain A. Role of Sphingosine-1-Phosphate in Mast Cell Functions and Asthma and Its Regulation by Non-Coding RNA. Front Immunol 2017;8:587.

(17) Vijayan M, Xia C, Song YE, Ngo H, Studstill CJ, Drews K, et al. Sphingosine 1-Phosphate Lyase Enhances the Activation of IKKepsilon To Promote Type I IFN-Mediated Innate Immune Responses to Influenza A Virus Infection. J Immunol 2017 Jul 15;199(2):677-87.

(18) Papadopoulos D, Rundle J, Patel R, Marshall I, Stretton J, Eaton R, et al. FTY720 ameliorates MOGinduced experimental autoimmune encephalomyelitis by suppressing both cellular and humoral immune responses. J Neurosci Res 2010 Feb 1;88(2):346-59.

(19) Penuelas-Rivas G, Dominguez-Perles R, Brinkmann V, Del Rio ML, Munoz-Luna A, Ramirez-Romero P, et al. FTY720 inhibits TH1-mediated allogeneic humoral immune response. Transplant Proc 2005 Nov;37(9):4124-6.

(20) Walsh KB, Marsolais D, Welch MJ, Rosen H, Oldstone MB. Treatment with a sphingosine analog does not alter the outcome of a persistent virus infection. Virology 2010 Feb 20;397(2):260-9.

(21) Subbarao K, Roberts A. Is there an ideal animal model for SARS? Trends Microbiol 2006 Jul;14(7):299303.

(22) Imai Y, Kuba K, Rao S, Huan Y, Guo F, Guan B, et al. Angiotensin-converting enzyme 2 protects from severe acute lung failure. Nature 2005 Jul 7;436(7047):112-6.

(23) Graham RL, Becker MM, Eckerle LD, Bolles M, Denison MR, Baric RS. A live, impaired-fidelity coronavirus vaccine protects in an aged, immunocompromised mouse model of lethal disease. Nat Med 2012 Dec;18(12):1820-6.

(24) Zhou P, Yang XL, Wang XG, Hu B, Zhang L, Zhang W, et al. A pneumonia outbreak associated with a new coronavirus of probable bat origin. Nature 2020 Mar;579(7798):270-3.

(25) Escobar LE, Molina-Cruz A, Barillas-Mury C. BCG vaccine protection from severe coronavirus disease 2019 (COVID-19). Proc Natl Acad Sci U S A 2020 Jul 28;117(30):17720-6.

(26) Lammers T, Sofias AM, van der Meel R, Schiffelers R, Storm G, Tacke F, et al. Dexamethasone nanomedicines for COVID-19. Nat Nanotechnol 2020 Aug;15(8):622-4.

(27) Oehrl S, Prakash H, Ebling A, Trenkler N, Wolbing P, Kunze A, et al. The phosphodiesterase 4 inhibitor apremilast inhibits Th1 but promotes Th17 responses induced by 6-sulfo LacNAc (slan) dendritic cells. J Dermatol Sci 2017 Aug;87(2):110-5.

(28) Maier C, Ramming A, Bergmann C, Weinkam R, Kittan N, Schett G, et al. Inhibition of phosphodiesterase 4 (PDE4) reduces dermal fibrosis by interfering with the release of interleukin-6 from M2 macrophages. Ann Rheum Dis 2017 Jun;76(6):1133-41.

(29) Klug F, Prakash H, Huber PE, Seibel T, Bender N, Halama N, et al. Low-dose irradiation programs macrophage differentiation to an iNOS(+)/M1 phenotype that orchestrates effective T cell immunotherapy. Cancer Cell 2013 Nov 11;24(5):589-602.

(30) Prakash H, Klug F, Nadella V, Mazumdar V, Schmitz-Winnenthal H, Umansky L. Low doses of gamma irradiation potentially modifies immunosuppressive tumor microenvironment by retuning tumor-associated macrophages: lesson from insulinoma. Carcinogenesis 2016 Mar;37(3):301-13. 
(31) Nadella V, Sharma L, Kumar P, Gupta P, Gupta UD, Tripathi S, et al. Sphingosine-1-Phosphate (S-1P) Promotes Differentiation of Naive Macrophages and Enhances Protective Immunity Against Mycobacterium tuberculosis. Front Immunol 2019;10:3085.

(32) Nadella V, Mohanty A, Sharma L, Yellaboina S, Mollenkopf HJ, Mazumdar VB, et al. Inhibitors of Apoptosis Protein Antagonists (Smac Mimetic Compounds) Control Polarization of Macrophages during Microbial Challenge and Sterile Inflammatory Responses. Front Immunol 2017;8:1792.

(33) Yu CC, Chien CT, Chang TC. M2 macrophage polarization modulates epithelial-mesenchymal transition in cisplatin-induced tubulointerstitial fibrosis. Biomedicine (Taipei) 2016 Mar;6(1):5.

(34) Zhang W, Ohno S, Steer B, Klee S, Staab-Weijnitz CA, Wagner D, et al. S100a4 Is Secreted by Alternatively Activated Alveolar Macrophages and Promotes Activation of Lung Fibroblasts in Pulmonary Fibrosis. Front Immunol 2018;9:1216.

(35) Broughton BR, Lim R, Arumugam TV, Drummond GR, Wallace EM, Sobey CG. Post-stroke inflammation and the potential efficacy of novel stem cell therapies: focus on amnion epithelial cells. Front Cell Neurosci 2012;6:66.

(36) Boxman IL, Ruwhof C, Boerman OC, Lowik CW, Ponec M. Role of fibroblasts in the regulation of proinflammatory interleukin IL-1, IL-6 and IL-8 levels induced by keratinocyte-derived IL-1. Arch Dermatol Res 1996 Jun;288(7):391-8.

(37) Hoegl S, Zwissler B, Eltzschig HK, Vohwinkel C. Acute respiratory distress syndrome following cardiovascular surgery: current concepts and novel therapeutic approaches. Curr Opin Anaesthesiol 2016 Feb;29(1):94-100.

(38) Chamoto K, Gibney BC, Ackermann M, Lee GS, Lin M, Konerding MA, et al. Alveolar macrophage dynamics in murine lung regeneration. J Cell Physiol 2012 Sep;227(9):3208-15.

(39) Myneni VD, McClain-Caldwell I, Martin D, Vitale-Cross L, Marko K, Firriolo JM, et al. Mesenchymal stromal cells from infants with simple polydactyly modulate immune responses more efficiently than adult mesenchymal stromal cells. Cytotherapy 2019 Feb;21(2):148-61.

(40) Spiller KL, Koh TJ. Macrophage-based therapeutic strategies in regenerative medicine. Adv Drug Deliv Rev 2017 Dec 1;122:74-83.

(41) Almatroodi SA, McDonald CF, Pouniotis DS. Alveolar Macrophage Polarisation in Lung Cancer. Lung Cancer Int 2014;2014:721087.

(42) Granata F, Frattini A, Loffredo S, Staiano RI, Petraroli A, Ribatti D, et al. Production of vascular endothelial growth factors from human lung macrophages induced by group IIA and group X secreted phospholipases A2. J Immunol 2010 May 1;184(9):5232-41. 